\title{
Frequency range size and the frequency range constraint in auditory perception by European starlings (Sturnus vulgaris)
}

\author{
STEWART H. HULSE, SUZANNE C. PAGE, and RICHARD F. BRAATEN \\ Johns Hopkins University, Baltimore, Maryland
}

\begin{abstract}
We report data bearing on the ability of European starlings to generalize a serial auditory pitch pattern discrimination from one frequency range to another. In earlier research (e.g., Hulse \& Cynx, 1985), European starlings failed to generalize such a discrimination from one 1-octave range of frequencies to another 1-octave range above or below the initial training range. The starlings thus demonstrated a hitherto unknown phenomenon, the frequency range constraint. In the present research, we first explored the frequency limits over which the range constraint might hold. The constraint was obtained after initial training in both a narrow (1/2-octave) and a wide (3-octave) training range. The latter range encompasses a substantial portion of the entire frequency range audible to starlings. Therefore, the constraint is independent of the size of the training range. No known principles of stimulus discrimination or generalization account for the constraint. We also obtained further evidence on starlings' sensitivity to relative pitch in auditory pattern perception. We used probe stimuli to explore pattern discrimination in narrow local regions of the wide frequency range. The probe data showed that, in spite of the frequency range constraint, the starlings were able to process pitch patterns on a relational basis. The results join earlier data indicating that both absolute and relative pitch processing may be involved in the natural auditory communication of songbirds.
\end{abstract}

In several recent experiments, European starlings (Sturnus vulgaris) have learned to discriminate between two sets of sound patterns. In one set, tones always rose in frequency; in the other, they always fell. Discrimination performance was then examined when the frequencies of pattern tones were shifted above, below, or within the range of frequencies in which the rising/falling discrimination had been learned initially (Cynx, Hulse, \& Polyzois, 1986; Hulse \& Cynx, 1985; Hulse, Page, \& Braaten, 1990).

Successful transfer to new frequencies was predicted on two assumptions. First, we assumed that the birds would perceive and learn the rising/falling frequency relationships in the original patterns. Then, we assumed that they would transfer the discrimination to new frequencies on the basis of those constant frequency relationships. That prediction was based on the fact that humans show a perceptual constancy for pitch relationships-the ups and downs in pitch, or the pitch contour, of familiar melodies. Perceptual constancy appears when melodies retain their identity with transpositions (changes) in key or pitch

The research reported in this paper was supported by National Science Foundation Research Grants BNS 8606307 and BNS 8911046, and by a National Science Foundation Graduate Fellowship to Suzanne C. Page. We thank Curtis Kline for his comments, Thomas J. Thomas for statistical advice, and Christian Grue of the Patuxent Wildlife Center, Laurel, MD, for supplying the starlings. Correspondence may be addressed to Stewart Hulse or Richard Braaten at the Department of Psychology, Johns Hopkins University, Baltimore, MD 21218 height (Deutsch, 1982). When such transpositions occur, absolute frequencies of the component tones of a melody shift substantially. However, pitch relations between neighboring tones remain the same, because-given the log scale of frequency upon which musical scales are built-frequency ratios between the tones remain the same. For these reasons, a familiar melody like "Yankee Doodle" maintains its identity when it is transposed from the key of $\mathrm{C}$ major to $\mathrm{G}$ major, or from a low pitch range on the piano keyboard to a high one. ${ }^{1}$

Although, for all the foregoing reasons, the starlings were predicted to transpose the rising/falling pitch discrimination, they failed to do so. In all cases, shifts of the rising/falling sequences to pitch ranges outside the familiar training range led to immediate loss of the discrimination-that is, to a failure to transpose and respond relationally. However, other tests showed that the birds could transfer the rising/falling discrimination to novel frequencies within the training range. Thus, failure to transfer was correlated only with shifts to novel frequencies outside a familiar range of frequencies, not to novel frequencies in general. For this reason, Cynx et al. (1986) named the phenomenon the frequency range constraint.

An experimental analysis of the frequency range constraint has shown-for starlings and for cebus monkeysthat the original pitch pattern discrimination was made mostly on the basis of memory for absolute stimulus features of the patterns, the absolute frequencies of their component tones in particular, and not on the basis of pitch 
relations (Cynx et al., 1986; D'Amato, 1988; Hulse \& Cynx, 1986; Page, Hulse, \& Cynx, 1989).

However, Hulse, Cynx, and Humpal (1984) and Page et al. (1989) found that starlings are capable of coding information on a relational basis under some conditions. For example, Page et al. showed that starlings could discriminate between a set of 32 exemplars that rose in pitch and another set of 32 exemplars that fell in pitch-even when absolute pitch features could not be used as a cue. However, they could only do so after preliminary training with a smaller set of exemplars in which either absolute or relative pitch cues were available as a cue. Page et al. (1989) and Hulse et al. (1990) have suggested that there may be a hierarchy of attentional or associative strategies such that processing on the basis of absolute frequency cues is preferred, but processing on the basis of relational information can be evoked as a secondary strategy. Although prior learning of an absolute pitch discrimination appears to play a role, the conditions that evoke the secondary relational strategy remain to be adequately identified.

The present research was undertaken with two purposes in mind. First, the frequency range constraint was examined when the training range for 4-tone rising and falling stimulus patterns was either a $1 / 2$ octave or 3 octaves in size. All the work to date has used training ranges in the neighborhood of 1 octave. Perhaps there is something unique about a 1-octave interval. Second, the experiment provided a new test for relational pitch processing in starlings.

Several facts are necessary to set the stage for our strategy and procedure. First, Hulse and Cynx (1985) showed that starlings readily generalize a rising/falling pitch pattern discrimination to novel frequencies as long as those frequencies remain within a familiar frequency range-namely, the range used in original baseline discrimination training. Second, Hulse and Cynx (1986) showed that starlings maintain a rising/falling pitch pattern discrimination in a familiar frequency range when the intervals between tones in the patterns are changed in size (e.g., from a whole tone to a semitone). Finally, with the go/no-go discrimination procedures that have been used in previous work, response latencies vary systematically as the frequencies contained in patterns within a familiar range move from one end of the range to the other (Hulse \& Cynx, 1986; Hulse et al., 1984; Hulse et al., 1990; Page et al., 1989). Short latencies result for high frequencies if birds are reinforced for pecks (go responses) to falling patterns (which generally begin on high frequencies in the range). Long latencies result for low frequencies if birds are trained to withhold pecks (nogo responses) for rising patterns (which generally begin on low frequencies in the range). Thus, a typical experiment shows a latency difference correlated with the discrimination between rising and falling patterns, but it also shows a systematic change in latency for both rising and falling patterns as their frequencies move from the low to the high end of the range. This is the absolute frequency gradient. In the example at hand, latencies become shorter in general as pattern frequencies move from low to high. The effect of absolute frequency may be sufficiently potent at the extremes of the frequency range to obscure any discrimination between rising and falling patterns (Hulse et al., 1984; Page et al., 1989).

In the present experiment, we capitalized on these three facts to provide a strong test for relational perception. After the starlings trained with the wide, 3-octave frequency range had learned the rising/falling pitch pattern discrimination, they were tested with probe patterns constructed with novel frequencies separated by the semitone intervals of the narrow, $1 / 2$-octave range. The probe patterns were placed between baseline frequencies at low, middle, and high frequency regions of the wide, 3-octave training range. If the starlings had learned nothing about relative pitch in the original rising/falling discrimination and were responding solely on the basis of some absolute frequency characteristics of the baseline patterns, they should respond with equal latencies to the rising and falling probe patterns. That would be so because all the tones of each probe pattern would be concentrated in just one narrow frequency region of the wide baseline range. Stimulus generalization within the region should assure virtually equivalent response latencies to all local frequencies within a narrow local region. Latencies to both rising and falling patterns might change from a low frequency region at one end of the 3-octave range to a high frequency region at the other, because of the absolute frequency gradient, but there should be no latency differences between rising and falling patterns within any given region.

If, on the other hand, the starlings had learned something about pitch relations in earlier training, they ought to maintain their differential behavior regardless of where probe patterns based on semitones were placed in the 3octave range. The change in interval size from 3 whole tones to 1 semitone should not affect the discrimination appreciably (Hulse \& Cynx, 1986), and all the novel probe patterns are within a familiar frequency range where starlings readily generalize a rising/falling pitch pattern discrimination (Hulse \& Cynx, 1985). In effect, the procedure pitted the starlings' secondary strategy to respond on a relational basis against their primary tendency to respond on the basis of absolute frequency. If relational responding were to occur under these circumstances, it would be additional convincing evidence that starlings can process relative pitch.

\section{METHOD}

\section{Subjects}

Six wild-caught naive starlings (Sturnus vulgaris), 3 male and 3 female adults of unknown age (Birds A26, A27, A28, A30, A31, and $\mathrm{M} 12$ ) were obtained from the Patuxent Federal Wild Life Preserve in Laurel, MD. Four additional male birds (Birds O20, P55, R10, and S54) were obtained from the same source and used in a replication of part of the experiment (see below). The starlings were maintained at $85 \%-90 \%$ of their free-feeding weight in in- 
dividual cages on a basic diet of Purina turkey starter, ground carrots, and molasses. The basic diet, combined with ground hardboiled eggs (including the shells) and moist dog food, served as the reinforcer. Grit and, on alternate days, water or a water-vitamin solution were available in the home cage. Water was available at all times in the test chamber. The light:dark cycle in the colony room was controlled to match the day:night cycle prevailing in Baltimore.

\section{Apparatus}

A detailed description of the apparatus appears in Hulse and Cynx (1985). Briefly, the starlings worked in a wire mesh test cage centered inside a sound-attenuating chamber. Pure sinusoidal tones were delivered through a speaker centered above the test cage. For birds in the narrow-range group, tones were generated by Apple II + microcomputers containing Mountain Computer music synthesizers (Mountain Computer, Scotts Valley, CA). Output from the synthesizers went to a Coulbourn Model \$84-04 rise/fall gate with a linear rise/fall time set for $5 \mathrm{msec}$. For birds in the wide-range group, pure sinusoidal tones were produced by a Krohn-Hite Model 4141R programmable oscillator feeding into a Grayson-Stadler Model 829D acoustic switch set for a rise and fall time of $5 \mathrm{msec}$. Output from the rise/fall gates was passed through an amplifier, and then sent to the chamber speaker.

The 4 starlings used in the replication were tested in an IAC soundattenuating chamber, Model AC3. Sinetones were generated digitally with a $20-\mathrm{kHz}$ sampling rate by an IBM Personal Computer AT, fed to Coulboum Model S84-04 rise/fall gates set for a linear rise/ fall time of $5 \mathrm{msec}$ and then to a Crown amplifier (Model DB7) and the speaker in the experimental chamber.

Three translucent response keys $2 \mathrm{~cm}$ in diameter were mounted $6 \mathrm{~cm}$ apart on a front panel in a horizontal row, $12 \mathrm{~cm}$ above the test cage floor. Pecks on each key were detected by a microswitch. A Gerbrands Model G5610 pigeon feeder was located below the center key.

In the original experiment, the Apple computers, programmable oscillator, experimental contingencies, and data recording were managed on line by a Digital Equipment Corporation PDP-8E minicomputer using the SuperSked operating system (State Systems, Kalamazoo, MI). In the replication, an IBM AT managed experimental contingencies and recorded data.

\section{Baseline Discrimination}

The baseline discrimination trained the starlings to discriminate rising from falling pitch patterns in either a $1 / 2$-octave or a 3-octave range.

Procedure. Procedures were identical for the original 6 starlings and for the $\mathbf{4}$ starlings run in the replication. To begin, naive starlings were autoshaped in a single session to peck the center and then the right key, and, finally, they were briefly reinforced for chained center $\rightarrow$ right response sequences.

The starlings were then trained on a go/no-go discrimination task similar to that described by Hulse et al. (1984). Trials began with a 5-sec intertrial interval followed by illumination of the center key. A peck to the center key darkened that key and initiated, with $p=.5$, either a rising or a falling tone pattern. Particular exemplars were drawn randomly (without replacement), from the stimulus set. After a 4-sec presentation period, during which the pattern repeated, the right key lit up. Sound stimuli continued for a maximum of 4 additional seconds. Pecks to the right key during the presentation period or pecks at any time to the center or left key had no consequence. A peck to the lit right key darkened that key, ended the pattern presentation, and, if the pattern was a rising sequence, produced 1.5-sec access to food. Pecks to the lit right key during a falling sequence resulted in a 10 -sec time-out. If no response occurred within 4 sec following illumination of the right key, keylights were darkened and the 5 -sec intertrial interval began. The houselight remained on at all times except during time-out.
Sessions were $3 \mathrm{~h}$ long and were conducted daily. Training continued until a starling had attained a criterion of at least a 2.5 -sec difference in response latency for rising versus falling patterns on 11 of 12 consecutive sessions. Then, a series of frequency transfer tests began.

Stimuli. During baseline training, the starlings were required to discriminate four 4-tone patterns that rose in frequency from four 4-tone patterns that fell in frequency. The pattern tones were sinusoids drawn from a list of frequencies that divided the frequency range into equal intervals on a log scale. This overall frequency range, centered at $1480 \mathrm{~Hz}$, was-for different birds-either $1 / 2$ octave or 3 octaves wide. In the $1 / 2$-octave training set, consecutive tones in a pattern were separated by a semitone. In the 3-octave training set, consecutive tones were separated by 3 whole tones.

The specific frequencies used to construct the patterns are displayed separately in Figure 1 for the $1 / 2$-octave and 3-octave ranges. The $1 / 2$-octave range spanned frequencies from 1245 to $1760 \mathrm{~Hz}$. Within the $1 / 2$-octave range, the four rising patterns began on 1245 , 1319,1397 , or $1480 \mathrm{~Hz}$ and rose 3 tones from there. A typical rising stimulus pattern might be $1319 \rightarrow 1397 \rightarrow 1480 \rightarrow 1568 \mathrm{~Hz}$. Similarly, four falling patterns began on $1760,1661,1568$, or $1480 \mathrm{~Hz}$ and descended 3 tones from there. Here, a typical falling stimulus pattern might be $1568 \rightarrow 1480 \rightarrow 1397 \rightarrow 1319 \mathrm{~Hz}$. The set of rising and falling patterns in the 3-octave range was constructed in the same way from the following frequencies: $523,740,1047,1480$, 2093,2960 , and $4186 \mathrm{~Hz}$. Only one pattern was presented on any given trial.

For both narrow- and wide-range stimuli, each tone in a pattern was $100 \mathrm{msec}$ long, and the intertone interval was $100 \mathrm{msec}$. A pattern repeated for a maximum of $8 \mathrm{sec}$, depending on experimental contingencies. Repetitions were separated by an $800-\mathrm{msec}$ interval. The patterns were formally similar in frequency contour and temporal structure to those used by Hulse et al. (1984). They differed only in tone frequency and in the size of the interval between successive tones.

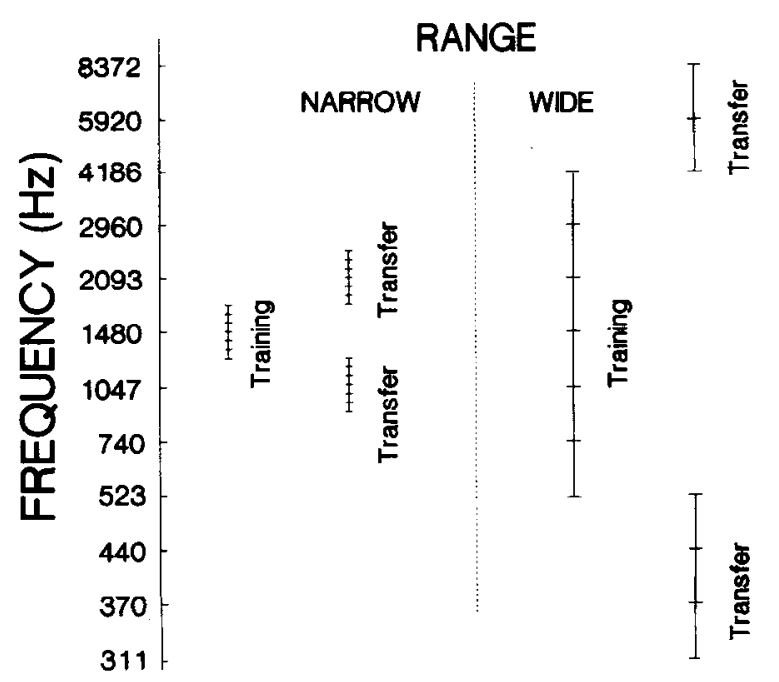

Figure 1. Frequencies, shown by horizontal hash marks, from which rising and falling pitch pattern stimuli were constructed for initial training and for transfers. The training stimuli were constructed from frequencies within a narrow $(1 / 2-\infty \mathrm{ctave})$ and a wide (3-octave) range. The transfers were to ranges above, within, or below the narrow and wide training ranges. The above-range transfers for the wide-range transfers were truncated to 3 instead of 4 tones, in order to keep the stimuli within the hearing range of the starlings. 
The tones used in constructing the sequences were set empirically at $55 \pm 5 \mathrm{~dB}(\mathrm{~A})$, as measured by a Rion Model NA-20 loudness meter with its microphone placed at the level of the response keys in the test chamber. Loudness levels were determined independently for each tone in each chamber and were recalibrated periodically. All tones were well within the starling's audibility range (Dooling, Okanoya, Downing, \& Hulse, 1986).

To ensure that the birds were not using loudness differences as a cue, a loudness transfer test was conducted for 4 birds, 3 from the wide-range condition and 1 from the narrow-range condition, following the completion of probe tests. ${ }^{2}$ The loudness test consisted of two sessions, during which each tone in a pattern was randomly set to $55,60,65$, or $70 \mathrm{~dB}(\mathrm{~A})$ and $50 \%$ of correct responses were reinforced. An analysis of variance revealed no significant difference in mean response latency between the last session prior to transfer and the first loudness transfer session $[F(1,3)=5.71$, $p>.05]$

\section{Transfer Tests for the Frequency Range Constraint}

Procedure. A series of three transfer tests immediately followed baseline training. Each transfer test consisted of two sessions with stimuli transposed to a new frequency range. Contingencies were identical to those used in training. Between each transfer test, the starlings returned to the baseline condition and to the stimulus frequencies used in initial training, and they remained there until the difference in mean response latency was $2.5 \mathrm{sec}$ or more for five consecutive sessions.

Stimuli. The stimulus frequencies used in the first and second transfer tests are also shown in Figure 1. In the first transfer, the stimuli were transposed to the frequency range immediately above the training range. In the second transfer, the stimuli were transposed to the frequency range immediately below the baseline training range. Finally, a within-range transfer test was conducted with stimuli transposed to novel frequencies within the baseline frequency range.

For the transfers above and below the baseline range, baseline frequencies were doubled and halved, respectively. For the withinrange transfer, frequencies were located halfway between (on a log scale) baseline frequencies. Because frequencies were located at equal intervals on a log scale, each transfer was a relational transposition.

Under both wide- and narrow-range conditions, three rising and three falling 4-tone patterns were constructed for the within-range transfers. For the narrow-range starlings, four rising and falling 4-tone patterns were used for transfers to both the above- and belowbaseline ranges. Because the pure-tone audibility curve for starlings begins to increase sharply at frequencies above $6 \mathrm{kHz}$ and below $0.5 \mathrm{kHz}$ (Dooling et al., 1986), only two patterns, one rising and one falling, were used to test whether or not wide-range birds would transfer the discrimination to new frequency ranges outside the baseline range. The test patterns were 4 tones long in the below-range transfer but were truncated to just 3 tones in the above-range transfer. Previous experiments done with similar training procedures have shown that performance is not impaired when patterns are truncated (Hulse \& Cynx, 1986; Hulse et al., 1984; Page et al., 1989). Apart from these differences, and except for the frequencies involved, the structure of the rising and falling patterns of the transposed stimuli was identical to that used in baseline training.

\section{Probe Tests for Relational Perception}

Following the completion of the transfer tests, the starlings in the wide-range group underwent a series of probe tests designed to test for relational perception. The starlings were exposed to rising and falling pitch pattern probes constructed from novel frequencies. The novel probes were placed in three regions. The high and low regions were located between two baseline frequencies at the extreme high and low ends of the baseline frequency range, respec-

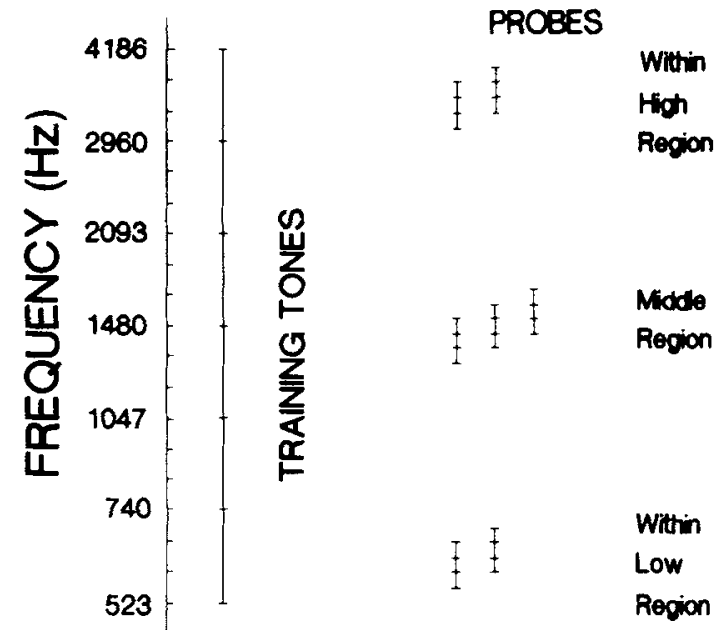

Figure 2. Probe-tone frequencies at the right, shown by horizontal hash marks, for the rising and falling pitch patterns used in the probe tests. A 4-tone rising probe pattern and a 4-tone falling probe pattern were constructed from the frequencies in each of the five probe sets. Probe frequencies within a pattern were spaced a semitone apart. For comparison purposes, frequencies from which rising and falling training patterns were constructed appear at left.

tively. The middle region included frequencies that spanned the mid dle of the baseline frequency range.

Comparable tests in the narrow, 1/2-octave range could not be conducted, because if probe patterns were constructed and located as those in the wide 3-octave range, frequency differences would not be discriminable to the starlings (Dooling et al., 1986).

Procedure. First, the probability that reinforcement would be available on trials in which a baseline rising sequence played was gradually reduced from 1.0 to 0.5 . Probe sessions began when the difference in mean latency to rising and falling sequences had exceeded $2.5 \mathrm{sec}$ for three consecutive baseline sessions.

In probe sessions, baseline stimuli occurred on a random $80 \%$ of all trials. For these trials, pecks during falling sequences produced time-out, and pecks during rising sequences produced reinforcement $(p=.5)$, as in training. Probe stimuli occurred on the remaining $20 \%$ of the trials. On probe trials, pecks merely terminated the trial and had no other consequence. Probe tests were conducted on consecutive days if the difference in mean latency to respond to baseline stimuli was at least $2.5 \mathrm{sec}$. In all other respects, the procedures were identical to those used for baseline discrimination training.

Stimuli. The frequencies used for the 4-tone rising and falling probe stimuli are shown in Figure 2 and Table 1. The probes incorporated frequencies that divided the interval between tones in the baseline wide-range stimuli into six equal steps on a log scale.

Table 1

Frequency Values (in Hertz) Used to Construct Probe Patterns in Low, Middle, and High Frequency Regions of the 3-Octave Range

\begin{tabular}{ccc}
\hline & Region & \\
\cline { 2 - 3 } Low & Middle & High \\
\hline 698 & 1710 & 3951 \\
659 & 1614 & 3729 \\
622 & 1523 & 3520 \\
587 & 1438 & 3322 \\
554 & 1357 & 3136 \\
\hline
\end{tabular}


Thus, successive notes in a probe were separated by a semitone. In the high region, rising and falling probe patterns consisted entirely of novel frequencies selected from frequencies in the range $3136-3951 \mathrm{~Hz}$. For example, the two highest frequencies in 3-octave baseline training were 2960 and $4186 \mathrm{~Hz}$. The frequencies of the high-region probe stimuli inserted into this interval were drawn from the list of five frequencies encompassing 3136-3951 Hz (Table 1). Thus, there were two rising probes and two falling probes. Similarly, there were two rising probes and two falling probes in the low region. Three probes were used in the middle region surrounding the middle training frequency, $1480 \mathrm{~Hz}$. Thus, the probes in the middle region did not lie completely within a training interval, but they did incorporate novel frequencies.

The probes from the high region specified in Figure 1 were tested first. The probes from the low region were used in a second probe test, and the probes from the middle region were used in a third and final probe test.

\section{RESULTS}

\section{Baseline Training}

All 10 starlings readily learned the initial baseline rising/falling pitch pattern discrimination in both the wide (3-octave) and narrow ( $1 / 2$-octave) baseline ranges. Analysis of variance for the last two baseline sessions prior to the first transfer test revealed a significant effect for

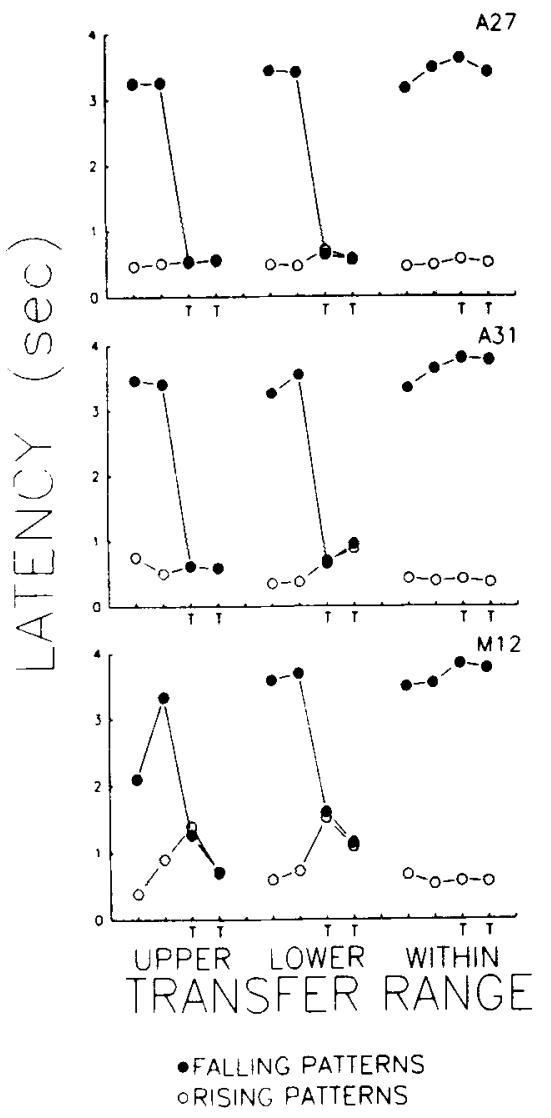

Figure 3. Mean baseline response latencies and mean latencies for transfers to new stimuli above, below, and within the baseline frequency range for starlings trained in the narrow frequency range. Data are presented for 2 baseline days prior to the transfer, and for the 1st and 2nd days of the transfer (both days marked $T$ ).

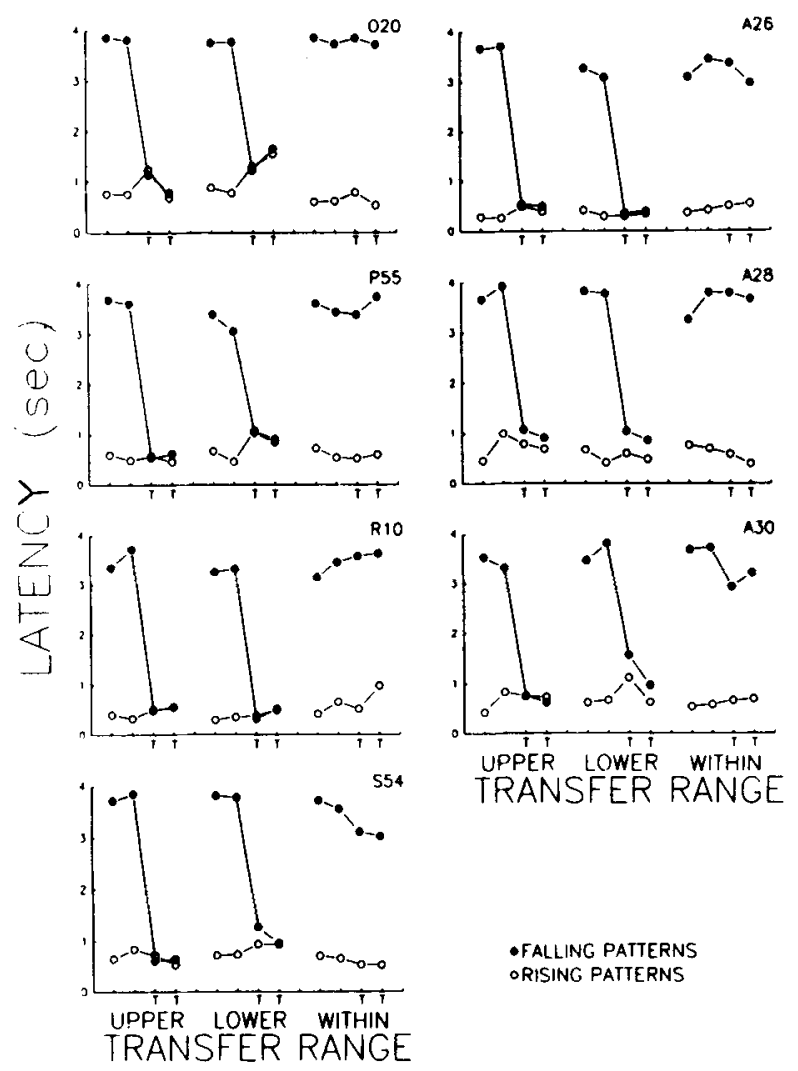

Figure 4. Mean baseline response latencies and mean latencies for transfers to new stimuli above, below, and within the baseline frequency range for starlings trained in the wide frequency range. Data are presented for 2 baseline days prior to the transfer, and for the 1st and 2nd days of the transfer (both days marked $T$ ).

pattern contour $[F(1,8)=349.16, p<.01]$. There were no significant differences between sessions, or between birds in the different training groups.

\section{The Frequency Range Constraint}

As Figures 3 and 4 show, all 7 starlings in the 3-octave training group and all 3 starlings in the $1 / 2$-octave training groups immediately lost the rising/falling discrimination when transferred to stimuli with frequencies outside the training range, regardless of whether the transposition was to higher or lower frequencies. That is, all birds exhibited the frequency range constraint.

As usual, however, all starlings maintained the rising/falling discrimination when transferred to stimuli composed of novel frequencies that remained within the range used in training. All birds also readily regained the discrimination when returned to baseline stimuli between the successive transfer tests.

Mean latencies to respond to rising and falling patterns in the session immediately before and immediately after transfer were compared in three separate analyses of variance. For transfers to both the upper and lower range, the interaction between pattern contour (whether patterns rose or fell in pitch) and session type (baseline or trans- 
fer) was significant [upper range transfer, $F(1,8)=$ $741.90, p<.01$; lower range transfer, $F(1,8)=$ $2,328.29, p<.01]$. Analysis of variance for mean latencies before and after transfer to new patterns within the training range yielded a significant effect for pattern contour $[F(1,8)=3,057.07, p<.001]$ but no effect of session type, and no interactions. Training condition, whether the birds were trained with patterns spanning $1 / 2$ octave or 3 octaves, was nonsignificant in all analyses and did not interact significantly with any other variable.

\section{Relational Perception}

As Figure 5 shows, evidence for relational perception appeared for most birds, especially for probes inserted into the middle region of the 3-octave training range. That is, latencies for falling probes were slower than latencies for rising probes. For 6 of the 7 starlings, the same result was obtained in the low region, although the latency differences were much smaller. There was little evidence of relational perception in the high region.

Figure 5 also shows a clear tendency in the birds to respond on the basis of absolute frequency. That is, there was a gradual change from short to long latencies as frequencies rose across the three probe ranges-regardless of whether probe stimuli rose or fell in pitch. This was true for most of the starlings, with the possible exception of Birds P55 (where latency differences were in the wrong direction) and R10 (where latency differences were small).

Analyses of variance on mean latencies collapsed across probes within the high, low, and middle regions showed significant effects for region $[F(2,12)=3.91, p<.05]$ and contour $[F(1,6)=11.82, p<.01]$, and for the interaction between the two $[F(2,12)=11.21, p<.01]$.

\section{DISCUSSION}

The starlings had little difficulty learning to distinguish rising from falling pitch patterns when tones in the patterns were separated by as much as 3 whole tones or by as little as a semitone. The data add to the abundance of earlier observations showing that songbirds maintain substantial flexibility in pitch pattern discrimination under many variations of rising/falling pitch patterns. For example, the discrimination appears in short, long, and truncated patterns (Hulse et al., 1984; Page et al., 1989) and for many different changes in interval size between adjacent pattern frequencies (Hulse \& Cynx, 1986). But all this is true only if pitch patterns remain within a familiar range of frequencies.

\section{Transfers to Unfamiliar Pitch Ranges and the Frequency Range Constraint}

The transposition data showed that the starlings immediately lost the rising/falling pitch discrimination-the frequency range constraint appeared-with shifts to unfamiliar frequencies outside the range in which the discrimination was originally learned. That was the case whether the initial training range was narrow ( $1 / 2$ octave)

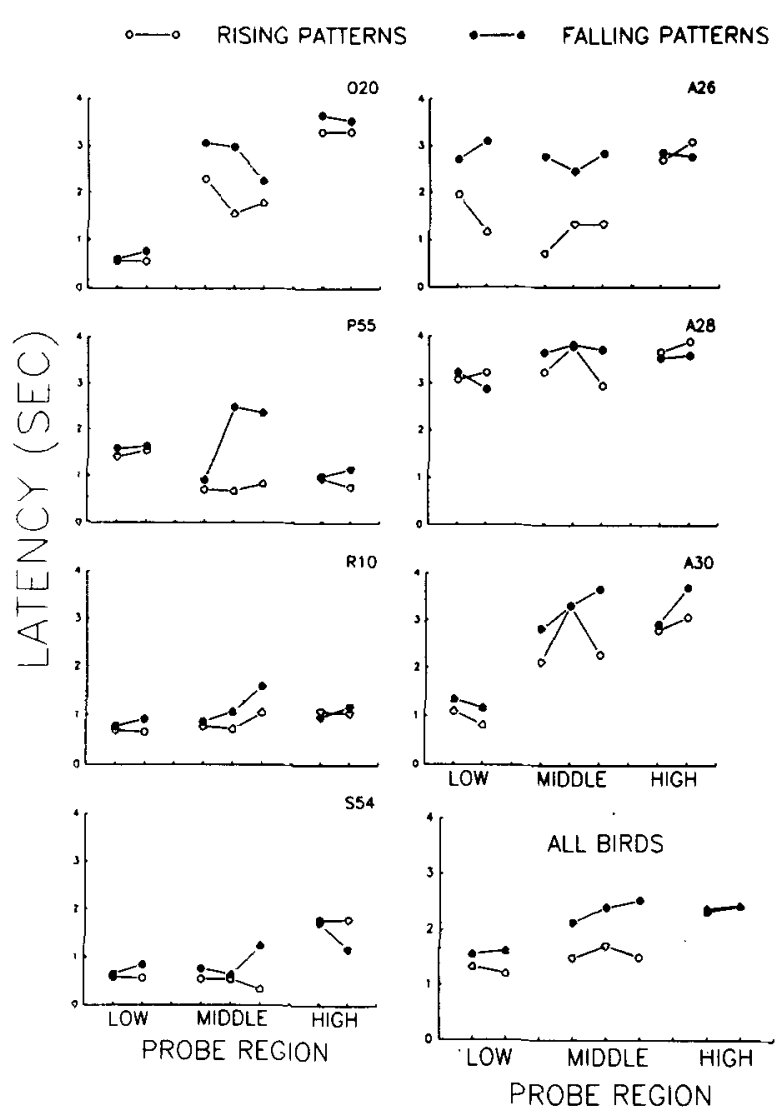

Figure 5. Mean response latencies for 7 starlings trained in the wide range to probe patterns that rose or fell in pitch in low, middle, and high regions of the baseline, wide-range stimuli. Mean latencies averaged across all birds appear at the lower right. For each bird, two probes were used in the low and high regions; three were used in the middle region. Within all regions, data are ordered from the left to right, according to the frequency of the initial tone of each probe pattern (cf. Figure 2).

or wide ( 3 octaves). These data extend earlier observations for 1-octave training ranges (Cynx et al., 1986; Hulse \& Cynx, 1985). Without doubt, the frequency range constraint is independent of range size.

However, starlings effortlessly transposed the discrimination to new frequencies within the familiar baseline range-whether that range was narrow or wide. This also replicates results from a number of earlier experiments (e.g., Cynx et al., 1986; Hulse \& Cynx, 1985). Again, successful within-range transfer is independent of the size of the range within which transfers occur.

Two important conclusions follow from these observations. First, the frequency range constraint and successful within-range transfer have now been demonstrated for frequencies that span virtually the entire frequency range to which starlings are sensitive. Second, the present baseline and transfer data are consistent with data from many earlier experiments-not only for starlings (e.g., Cynx et al., 1986; Hulse et al., 1984), but also for mockingbirds and cowbirds (Hulse \& Cynx, 1985), and for cebus monkeys (D'Amato, 1988). They thus assure that data 
from the probe stimuli, to which we now turn, rest on a valid, well-established foundation.

\section{Probes in Narrow Pitch Ranges: \\ Relational and Absolute Perception}

As before, evidence was obtained for both relative and absolute pitch processing by the starlings when novel, narrow-range probe patterns were inserted into localized regions of the wide, 3-octave range. Of the 7 birds tested, all showed relative pitch processing-especially in the middle region of the range. In each case, the birds were able to maintain the rising/falling discrimination. If the birds were responding solely on the basis of absolute frequency cues, latencies should have been virtually identical within all regions for rising and falling patterns, because both rising and falling probes consisted of tones of very similar pitch when compared with the full span of the 3-octave range.

The clearest case of absolute pitch processing in the sense that good discrimination was tied to some form of absolute frequency cue comes, of course, from the frequency range constraint. However, absolute pitch processing also appeared when birds gave short-latency responses, in general, in the low region and long-latency responses, in general, in the high region. That pattern of behavior was evident for most of the starlings (cf. Figure 5). This outcome, the absolute frequency gradient, has been obtained before (e.g., Cynx et al., 1986; Hulse et al., 1984; Page et al., 1989).

\section{Implications for Within-Range Transfers}

The foregoing leads to an important new conclusion about processes underlying within-range transfers. The probe data of the present experiment show that starlings discriminated very well among rising and falling pitch patterns in very narrow frequency regions of a familiar 3octave range. They did this when, on the basis of stimulus generalization, they should not-because all the frequencies of the probe patterns within the high, middle, or low regions were close together in a narrow band. It follows that excellent performance in within-range transfers, which has been obtained before (Hulse \& Cynx, 1985), cannot be based solely on stimulus generalization across familiar absolute frequencies. Instead, it must be based at least in part on relational pitch processing.

\section{CONCLUSIONS}

It is truly remarkable that pitch pattern processing is so completely bounded by the frequency range constraint. The phenomenon has now been demonstrated under a variety of stimulus conditions and for species as different as songbirds and nonhuman primates (D'Amato, 1988). No known principle of stimulus generalization or concept formation can account for the phenomenon; it is a simple tautology to say that the phenomenon results from a failure of stimulus generalization or from the failure to transpose a relational concept. Furthermore, there is nothing in adult human pitch perception that appears comparable. It is commonly held-especially among musicians-that human adults maintain perceptual constancy for transpositions of pitch patterns from one frequency region to another. In fact, however, experiments with adult humans have yet to be done with procedures directly paralleling ours.

Some evidence from children is very intriguing, however. McKee and Riley (1962), Riley and McKee (1963), and Riley, McKee, and Hadley (1964) found that first-grade (6-year-old) children, third-grade children, and college students could readily transfer a rising/falling auditory discrimination if loudness was the relevant dimension, but not if pitch was the relevant dimension. Pretraining in ordering auditory stimuli according to frequency facilitated transposition to some extent for third-graders (first-graders and college students were not tested under this condition), but still transposition was far from perfect. These data parallel those we have obtained with songbirds. Trehub (1987), on the other hand, summarizes evidence from infants which suggests that newborns can process pitch patterns relationally. Infants, in a habituation task, sometimes failed to notice transpositions of pitch patterns across pitch height, which suggests that they perceived the transposed patterns to be the same as the original patterns and, thus, maintained perceptual constancy. The evidence is not clear-cut on this issue, however, and the data Trehub discusses, and her conclusions, are not in line with those of Riley and McKee. Nevertheless, further experiments involving relative and absolute pitch, with subjects who are more or less naive with respect to pitch processing, would be useful.

Experiments are also needed to find out how to turn a novel, unfamiliar pitch range into one that is sufficiently familiar to support transfer of relational pitch information, and how this necessary experience differs across age and species. Riley and McKee's experiments are a step in this direction. For older humans, simple exposure to different pitches may be sufficient, whereas younger humans may need to prelearn pitch orderings. We have recently suggested that songbirds process pitch patterns according to a hierarchy of processing strategies (Braaten, Hulse, \& Page, in press; Hulse \& Page, 1988; Hulse et al., 1990; Page et al., 1989). This hierarchical model may provide clues about the conditions that are necessary to facilitate the transfer of relational pitch processing to novel frequency ranges. According to the model, absolute pitch processing lies at the bottom of the hierarchy. Ordinal discrimination ("higher than" and "lower than" classification) among isolated pitches is the next most complex level in the hierarchy. Discrimination among pitch pattern contours, where pitches unfold successively in time, lies at the top of the hierarchy. The evidence suggests that starlings learn the hierarchy from the bottom up. Riley et al.'s (1964) evidence suggests the same for humans. The model should provide further insights into cross-species and developmental considerations, among other things, for the matter at hand. 
For the time being, we are left to observe that the frequency range constraint, quite apart from its theoretical explanation, has important implications for the mechanisms that nonhuman animals may invoke when they process serial patterns of auditory information. This is so not only for artificial sound patterns in the laboratory or elsewhere, but also for the interpretation of natural auditory events. There is growing recognition that both absolute and relative frequency perception play a role in auditory communication (see, e.g., Becker, 1982; Hill \& Lein, 1987; Nowicki, 1983; Park \& Dooling, 1985; Ratcliffe \& Weisman, 1985, 1987; Weisman \& Ratcliffe, 1987, 1989).

\section{REFERENCES}

Becker, P. H. (1982). The coding of species-specific characteristics in bird sounds. In D. E. Kroodsma \& E. H. Miller (Eds.), Acoustic communication in birds (Vol. 1, pp. 214-252). New York: Academic Press.

Braaten, R. F., Hulse, S. H., \& Page, S. C. (in press). Discrimina tion and classification of rising and nonrising pitch patterns by the European starling (Sturnus vulgaris). Animal Learning \& Behavior.

CynX, J., Hulse, S. H., \& Polyzors, S. (1986). A psychophysical measure of pitch discrimination loss resulting from a frequency range constraint in European starlings (Stumus vulgaris). Journal of Experimental Psychology: Animal Behavior Processes, 12, 394-402.

D'Amato, M. R. (1988). A search for tonal pattern perception in cebus monkeys: Why monkeys can't hum a tune. Music Perception, $5,453-480$.

Deursch, D. (Ed.) (1982). The psychology of music. New York: Academic Press.

Dooling, R. J., Okanoya, K., Downing, J., \&ulse, S. [H.] (1986). Hearing in the starling (Stumus vulgaris): Absolute thresholds and critical ratios. Bulletin of the Psychonomic Society, 24, 462-464.

GreEN, D. M. (1976). An introduction to hearing. Hillsdale, NJ: Erlbaum.

HILL, B. G., \& LEIN, M. R. (1987). Function of frequency-shifted songs of black capped chickadees. Condor, 89, 914-915.

Hutse, S. H., CYNX, J. (1985). Relative pitch perception is constrained by absolute pitch in songbirds (Mimus, Molothrus, and Stumus). Journal of Comparative Psychology, 99, 176-196.

HuLSE, S. H., \& CYNX, J. (1986). Interval and contour in serial pitch perception by a passerine bird, the European starling (Stumus vulgaris). Journal of Comparative Psychology, 100, 215-228.

Hulse, S. H., CYNX, J., \& Humpal, J. (1984). Absolute and relative pitch discrimination in serial pitch perception by birds. Journal of $E x$ perimental Psychology: General, 113, 38-54.
Hulse, S. H., PAGE, S. C. (1988). Toward a comparative psychology of music perception. Music Perception, 5, 427-452.

Hulse, S. H., Page, S. C., Branten, R. F. (1990). An integrative approach to serial patern learning, music perception, and comparative acoustic perception. In M. Berkley \& W. C. Stebbins (Eds.), Comparative perception (Vol. 2, pp. 3-34). New York: Wiley.

MCKeE, J. P., RILEY, D. A. (1962). Auditory transposition in sixyear-old children. Child Development, 33, 469-476.

NowICKI, S. (1983). Flock-specific recognition of chickadee calls. Behavioral \& Ecological Sociobiology, 12, 317-320.

PAGE, S. C., Hulse, S. H., CYNX, J. (1989). Relative pitch perception in the European starling (Stumus vulgaris): Further evidence for an elusive phenomenon. Joumal of Experimental Psychology: Animal Behavior Processes, 15, 137-146.

Park, T., Dooling, R. J. (1985). Perception of species-specific contact calls by budgerigars (Melopsittacus undulatus). Joumal of Comparative Psychology, 99, 391-402.

RATClifFe, L., WeIsmaN, R. G. (1985). Frequency shift in the fee bee song of the black-capped chickadee. Condor, 87, 555-556.

Ratcliffe, L., Weisman, R. G. (1987). Phrase order recognition by brown-headed cowbirds. Animal Behavior, 35, 1260-1262.

Riley, D. A., McKee, J. P. (1963). Pitch and loudness transposition in children and adults. Child Development, 34, 471-482.

Riley, D. A., McKee, J. P., Hadley, R. W. (1964). Prediction of auditory discrimination learning and transposition from children's auditory ordering ability. Journal of Experimental Psychology, 67, 324-329.

TREHUB, S. E. (1987). Infants' perception of musical patterns. Perception \& Psychophysics, 41, 635-641.

WessmaN, R. G., \& RATCLIFFE, L. (1987). How birds identify species information in song: A pattern recognition approach. Learning \& Motivation, $18,80-98$.

Weisman, R. G., RAtCliffe, L. (1989). Absolute and relative pitch processing in black-capped chickadees (Parus atricapillus). Animal Behavior, 38, 685-692.

\section{NOTES}

1. Many acoustic factors can produce the psychological experience of pitch, and there are a corresponding number of theoretical accounts for the perception of pitch (Green, 1976). Throughout this paper, we adopt the operational definition that the pitch of a tone is defined by the frequency of a matching sine wave.

2. The experiment was internupted before the completion of the loudness transfers when the laboratory moved to new quarters. All 3 birds in the long-range group, but only 1 bird from the short-range group, completed the loudness test.

(Manuscript received September 30, 1988; revision accepted for publication February $23,1990$. ) 